\title{
Multiple Triangulation Analysis: another approach to determine the orientation of magnetic flux ropes
}

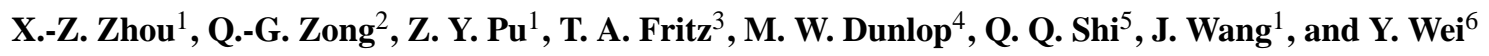 \\ ${ }^{1}$ Institute of Space Physics and Applied Technology, Peking University, Beijing 100871, China \\ ${ }^{2}$ Center for Atmospheric Research, University of Massachusetts, Lowell, MA 01854, USA \\ ${ }^{3}$ Center for Space Physics, Boston University, Boston, MA 02215, USA \\ ${ }^{4}$ Space Sciences Division, Rutherford Appleton Laboratory, Oxfordshire, UK \\ ${ }^{5}$ Center for Space Science and Applied Research, Chinese Academy of Sciences, Beijing 100080, China \\ ${ }^{6}$ Institute of Geology and Geophysics, Chinese Academy of Sciences, Beijing 100029, China
}

Received: 10 January 2006 - Revised: 18 April 2006 - Accepted: 17 May 2006 - Published: 3 July 2006

\begin{abstract}
Another approach (Multiple Triangulation Analysis, MTA) is presented to determine the orientation of magnetic flux rope, based on 4-point measurements. A 2-D flux rope model is used to examine the accuracy of the MTA technique in a theoretical way. It is found that the precision of the estimated orientation is dependent on both the spacecraft separation and the constellation path relative to the flux rope structure. However, the MTA error range can be shown to be smaller than that of the traditional MVA technique. As an application to real Cluster data, several flux rope events on 26 January 2001 are analyzed using MTA, to obtain their orientations. The results are compared with the ones obtained by several other methods which also yield flux rope orientation. The estimated axis orientations are shown to be fairly close, suggesting the reliability of the MTA method.
\end{abstract}

Keywords. Magnetospheric physics (Magnetopause, cusp and boundary layers; Solar wind-magnetosphere interactions; Instruments and techniques)

\section{Introduction}

Many techniques have been developed to study the shape and motion of a certain structure, by analyzing in situ data from single or multiple spacecraft. Siscoe et al. (1968) made use of the magnetic field measurements to obtain the normal direction of a tangential discontinuity. A more generalized approach was employed by Sonnerup and Cahill Jr. (1968) to fit the cases of both rotational and tangential discontinuities, as the very well-known method of Minimum Variance Analysis (MVA). Recently, some other methods, such as Minimum Faraday Residue (MFR) and Minimum Massflux Residue (MMR) were established, based on the conservation of tangential electric field and the mass flux through-

Correspondence to: $\mathrm{Z}$. Y. $\mathrm{Pu}$

(zypu@pku.edu.cn) out the current layer, respectively (Khrabrov and Sonnerup, 1998b; Sonnerup et al., 2004).

An example of multi-spacecraft methods is the triangulation method, also frequently referred to as the "timing method", based on the time differences between four spacecraft encountering the same structure (Russell et al., 1983). All of these techniques can be either used alone, or combined with other methods, to accurately determine the detailed properties of a certain 1-D structure (e.g. Haaland et al., 2004a).

However, these methods cannot be automatically applied to more complicated structures, e.g. flux ropes. It is widely accepted that the flux ropes can be treated as cylindrical 2-D structures with twisted magnetic field lines. Many flux rope events at the high-latitude magnetopause region have been observed by Cluster satellites (e.g. Zong et al., 2003; Pu et al., 2005). However, there remains a problem on how to determine their axial orientation via satellite data.

Elphic and Russell (1983) suggested a method called Principal Axis Analysis (PAA), to judge the axial orientation of flux ropes when they tried to explore the Venus ionosphere. The idea was based on the MVA technique (Sonnerup and Cahill Jr., 1968), to seek the three directions of maximum, intermediate and minimum magnetic field variances (marked as $\mathrm{L}, \mathrm{M}$ and $\mathrm{N}$ directions). However, in many cases the three directions cannot be distinguished clearly because of the similarity of the corresponding three magnetic field variances. Furthermore, it was concluded by Xiao et al. (2004) that the axial orientation could be in the $\mathrm{L}, \mathrm{M}$ and even $\mathrm{N}$ directions of the PAA technique, depending on different flux rope models: (1) M direction in force-free model (Lepping et al., 1990); (2) M or L directions in non-force-free models, with an intense core field suggested by Elphic and Russell (1983); (3) N direction in the "Grazing" FTE model by Farrugia-Elphic-Southwood (Farrugia et al., 1987; Elphic and Southwood, 1987). In order to avoid the uncertainties of the PAA technique, Xiao et al. (2004) suggested another

Published by Copernicus GmbH on behalf of the European Geosciences Union. 


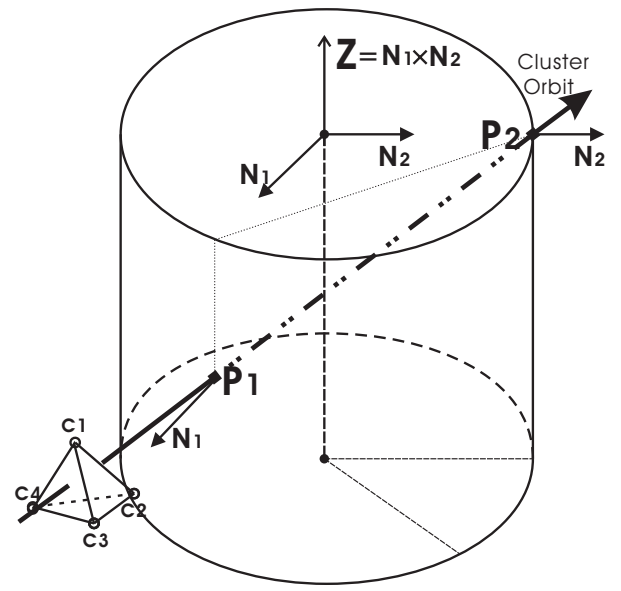

Fig. 1. Sketch of the Cluster constellation passing through a flux rope, which meets with the certain magnetic contour plane twice, at the positions of $P_{1}$ and $P_{2}$. Using the Triangulation Method, the normal directions of them can be obtained, marked as $N_{1}$ and $N_{2}$, both of which have no $z$ components. Thus, the cross product of them should point to the flux rope axis.

method (CMVA, or MVAJ) using the current instead of the magnetic field to perform MVA analysis, which was proven to highly enhance the accuracy of the axial orientation if reliable current data were available. The four Cluster magnetic field data sets allow for a calculation of curl B (Dunlop et al., 1988) which provides an estimate of the current density. The current-based MVA technique (also developed by Haaland et al., 2004b), however, sometimes gives large errors when the calculation of the current is not reliable.

The axial orientation of flux ropes can also be obtained as a byproduct of a technique called Grad-Shafranov (GS) Reconstruction (e.g. Hau and Sonnerup, 1999; Hu and Sonnerup, 2002). The technique offers a substantial field of view of the region around the trajectory of a certain spacecraft by solving the Grad-Shafranov equation, which arises from the stationary, 2-D form of Faraday's law. The method can be very accurate in determining the flux rope orientation. However, there is a strong limitation that the convective inertia terms must be negligible in a proper frame, i.e. the transverse component of the plasma flow velocity in the frame must be much smaller than the Alfvén velocity and the sound speed, which does not happen in cases with notable reconnection signatures.

A technique called Minimum Directional Derivative (MDD) method, based on 4-spacecraft magnetic field observations and the associated magnetic spatial gradients, recently suggested by Shi et al. (2005), can provide another choice to determine the flux rope orientation, through determination of the invariant axis. The method estimates the three principle directions for the directional derivatives point by point in time. If the direction of minimum derivative (gra- dient) is remarkably smaller than the other two directional gradients, the magnetic structure is interpreted as a 2-D structure and the direction obtained should agree with the flux rope orientation.

In this paper, another approach is established to judge the axial orientation of flux ropes, namely, Multiple Triangulation Analysis (MTA), which is developed from the Triangulation Method (or Timing Method) (Russell et al., 1983). We will theoretically examine the accuracy of the MTA approach within models, apply the technique to the real Cluster data sets and then compare the results with those obtained by other methods (MDD and CMVA), in order to confirm the effectiveness of the MTA technique.

\section{The MTA technique}

The Triangulation Method was initially presented to measure the interplanetary shock normal direction (Russell et al., 1983). The shock is assumed to be planar and the relative motion to the constellation is assumed to be constant. Its normal direction can be determined by solving the following equations: $\left(\boldsymbol{P}_{\boldsymbol{n}}-\boldsymbol{P}_{\mathbf{1}}\right) \cdot \boldsymbol{N}=V\left(t_{n}-t_{1}\right)$, where $\boldsymbol{P}_{\boldsymbol{n}}$ and $t_{n}$ represent the shock-arriving positions and times of the $n$-th satellites, $\boldsymbol{N}$ shows the direction of the shock normal and $V$ is the normal component of the moving velocity.

Choosing the magnetic field magnitude as the signal, we may apply the above method to the flux rope cases to calculate the normal directions of any magnetic contour planes inside the flux rope. A generalized flux rope model is adopted, with the magnetic topology being expressed as $|B|=f(\rho, \phi)$ in a cylindrical coordinate system, indicating the magnetic strength independency on $z$ values. Here the $\rho, \phi$ and $z$ axis are corresponding to the radial, azimuthal and axial directions, respectively.

For such a 2-D model, the gradient of magnetic field strength within the flux rope has only $\rho$ and $\phi$ components, suggesting the normal directions of all the magnetic contour planes to be perpendicular to the $z$ direction, i.e. the axial orientation of the flux rope. Because the Cluster constellation would basically pass through a certain contour plane twice (arriving and leaving), two normal directions can be measured. If they are not exactly in the same direction, the cross product of them should be pointing to the axial direction of the flux rope, as is shown in Fig. 1.

Random errors may occur because of the measurement inaccuracy and even the quantization effects in applying the timing method. In order to get rid of the effect of these errors, we may further select several contour planes with different magnetic strength and then calculate their normal directions, say, $\boldsymbol{N}^{(\boldsymbol{m})}(m=1, M)$, respectively. Each pair will, in principle, give an estimate of the axial direction (although somewhat dependent on the actual magnetic structure in the flux tube) and a mean value can be found. Theoretically, however, the flux rope axial direction $N_{\mathbf{0}}$ should be perpendicular 
to the normal vectors individually, so that in practice, the $\boldsymbol{N}_{\mathbf{0}}$ can be determined by the minimization of

$\sigma^{2}=\frac{1}{M} \sum_{m=1}^{M}\left|N^{(m)} \cdot N_{\mathbf{0}}\right|^{2}$.

The principle of the minimization method has been used in Siscoe et al. (1968), and it is also very similar to the one used in the well-known MVA technique (in detail, see Sonnerup and Scheible, 1998): introducing a Lagrange multiplier $\lambda$ and then seeking the solution of a set of three homogeneous linear equations in the normalization constraint of $N_{\mathbf{0}}^{2}-1=0$. Thus, the minimization becomes a problem of calculating the three eigenvalues $\left(\lambda_{1}, \lambda_{2}, \lambda_{3}\right.$, in order of decreasing magnitude) and corresponding eigenvectors $\left(\boldsymbol{x}_{\mathbf{1}}, \boldsymbol{x}_{\mathbf{2}}, \boldsymbol{x}_{\mathbf{3}}\right)$ of a symmetrical matrix

$L_{\mu \nu}=\frac{1}{M} \sum_{m=1}^{M} N_{\mu}^{(m)} N_{\nu}^{(m)}$,

where the subscripts $\mu, v=1,2,3$ denote the Cartesian components of the vector $N^{(m)}$ along the X, Y, Z system, respectively. Among the three eigenvectors, $\boldsymbol{x}_{\mathbf{3}}$ along with the smallest corresponding eigenvalue $\lambda_{3}$ can be used as the estimator for the flux rope axial orientation and $\lambda_{3}$ itself represents the estimating precision of the MTA technique. The smaller the $\lambda_{3}$ than the other two eigenvalues $\left(\lambda_{1}\right.$ and $\left.\lambda_{2}\right)$, the higher the reliability of the estimation.

Since the minimization methods used here and the one in minimum variance analysis are very alike, with only a little difference on the format of the symmetrical matrix, the angular standard deviation of the resulting MTA flux rope orientation can be obtained, following a procedure very similar to the MVA error estimation process (Khrabrov and Sonnerup, 1998a). The procedure (see Appendix in more detail) shows that the deviation can be expressed as the function of the three eigenvalues $\left(\lambda_{1}, \lambda_{2}, \lambda_{3}\right)$ and the number of normal vectors obtained by the Triangulation method $(M)$ :

$\Delta \phi_{i}=\sqrt{\frac{\lambda_{3} \lambda_{i}}{M\left(\lambda_{i}-\lambda_{3}\right)^{2}}}, \quad i=1,2$,

where $\Delta \phi_{i}$ represents the standard deviation (in radians) of the resulting $\boldsymbol{x}_{\mathbf{3}}$ toward the other two eigenvectors. Besides indicating the effect of eigenvalue separation on the MTA accuracy, it can also be seen that a large $M$ value, i.e. more contour planes selected for determining the normal directions, would effectively reduce the statistical errors of MTA technique.

The estimation error may also come from the non-planar properties of magnetic contour planes within the flux rope, as a systematic error. Since the MTA technique is based on the Triangulation method with an assumption that the plane is planar, the validity of MTA thus requires the spacecraft separations to be much smaller than the spatial scale of the flux rope, or the curvature radius of the magnetic contour plane
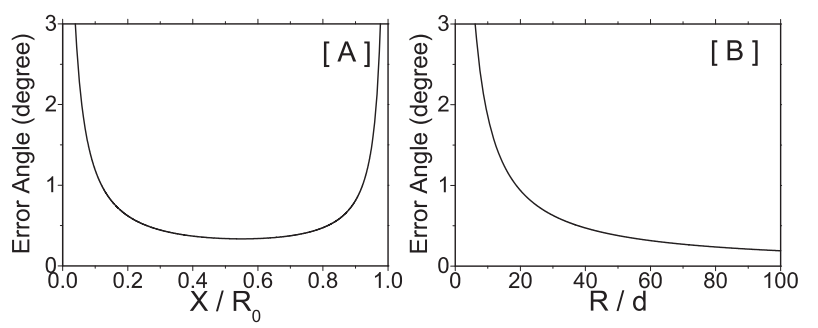

Fig. 2. The error range obtained by MTA tests, selecting only one contour plane in the certain flux rope model. The error is defined as the angle between the model $z$ axis and the MTA resulting axial orientation. (a) The error as a function of $X$ (the constellation's closest distance to the axis, normalized to flux rope radius $R$ ), when $R$ is 50 times greater than the satellites' separation $d$. (b) The error versus flux rope radius $R$ (normalized to $d$ ) in a certain type of Cluster trajectory with $X$ to be $0.707 R$.

where the constellation traverses the flux rope. As an error estimation, a set of MTA tests are performed to calculate the error range by calculating the angle between the model $z$ axis and the MTA result. For simplicity, the cross section of the flux rope is assumed to be circular with a radius of $R$, the 4spacecraft Cluster formation to be a regular tetrahedron with a distance of $d$ between each two satellites, and we select only one contour plane in each of the tests to obtain the flux rope orientation as the cross product of the two normals. It is found that the error depends not only on the flux rope radius, but also on the path that the satellites pass through the structure. The error dependencies on both $R$ and the Cluster passing path (characterized by the closest distance $X$ from the constellation to the axis) are clearly displayed in Fig. 2.

Figure 2a shows the resulting MTA error as a function of $X$ (normalized by $R$ ), in the typical case of the flux rope radius $R$ being 50 times as $d$. It can be seen that the error angle is very small (less than $3 \mathrm{deg}$ ) in the region of $0.04 R<X<0.98 R$. For those $X>0.98 R$ cases, the relatively larger error can be naturally understood to be caused by the constellation's skimming motion over the flux rope, however, the error for those $X<0.04 R$ cases is a bit more amazing. Actually, in the cases when the satellites almost pass through the axis of the flux rope, the two normal directions obtained at the positions of $P_{1}$ and $P_{2}$ are almost parallel, so that the cross product process may become invalid, leading to unexpected errors. However, if we select more contour planes in these cases, the resulting minimum eigenvalue $\lambda_{3}$ should be very close to the intermediate one, $\lambda_{2}$, indicating the approximate parallelism of all these normal directions, and providing a warning of the invalidation of the MTA technique in those cases.

The error dependency on flux rope radius $R$ (normalized by $d$ ) is also shown in Fig. 2 b, with a certain constellation trajectory, with $X / R$ equal to 0.707 . It is very clear that the error strongly depends on the ratio of flux rope radius $R$ to 
Table 1. The results of the Triangulation method as the first step of MTA: $|B|$ or $B_{N}$ selected as the signals, timings input and the normals obtained.

\begin{tabular}{lcc}
\hline$|B|$ & Timings of the 4 spacecrafts(s) & Normal Obtained \\
\hline $33 \mathrm{nT}$ & $34.28,31.95,31.16,32.30$ & $(-0.647,-0.162,0.745)$ \\
$34 \mathrm{nT}$ & $34.61,32.43,31.82,32.66$ & $(-0.600,-0.104,0.793)$ \\
$35 \mathrm{nT}$ & $35.02,32.89,32.34,33.03$ & $(-0.562,-0.073,0.824)$ \\
$36 \mathrm{nT}$ & $35.54,33.33,32.68,33.42$ & $(-0.552,-0.091,0.829)$ \\
$37 \mathrm{nT}$ & $35.99,33.78,33.01,33.90$ & $(-0.579,-0.136,0.804)$ \\
\hline $33 \mathrm{nT}$ & $49.84,47.99,47.58,47.90$ & $(-0.450,-0.002,0.893)$ \\
$34 \mathrm{nT}$ & $48.74,46.93,46.35,46.68$ & $(-0.416,-0.048,0.908)$ \\
$35 \mathrm{nT}$ & $47.24,45.41,45.17,45.99$ & $(-0.729,-0.062,0.682)$ \\
$36 \mathrm{nT}$ & $46.05,44.27,44.07,44.93$ & $(-0.764,-0.066,0.641)$ \\
$37 \mathrm{nT}$ & $44.75,43.05,42.90,44.02$ & $(-0.877,-0.116,0.465)$ \\
\hline$B_{N}$ & Timings of the 4 spacecrafts(s) & Normal Obtained \\
\hline $2 \mathrm{nT}$ & $39.52,37.33,36.54,36.68$ & $(-0.307,-0.025,0.951)$ \\
$4 \mathrm{nT}$ & $38.82,36.72,36.12,36.28$ & $(-0.342,0.001,0.940)$ \\
$6 \mathrm{nT}$ & $38.40,36.25,35.67,35.86$ & $(-0.358,0.004,0.934)$ \\
$8 \mathrm{nT}$ & $38.03,35.87,35.27,35.51$ & $(-0.374,-0.007,0.927)$ \\
$10 \mathrm{nT}$ & $37.60,35.41,34.86,35.15$ & $(-0.403,-0.002,0.915)$ \\
$12 \mathrm{nT}$ & $36.91,34.90,34.47,34.81$ & $(-0.450,0.003,0.893)$ \\
\hline
\end{tabular}

satellites' separation distance $d$ as the prediction, with the error angle less than $3 \mathrm{deg}$ in the case of $R$ greater than $6.1 \mathrm{~d}$.

As a comparison with previous methods, we may have a look at the error range produced by other techniques. Xiao et al. (2004) concluded that the errors of PAA and CMVA are strongly dependent on models and the satellite paths, with a typical error of around 15-20 deg. The possible error range of the MDD technique was also tested (Shi et al., 2005), using the flux rope model by Elphic and Russell (1983). They drew the conclusion that the angle between the calculated axis and the real axis is mostly less than $5 \mathrm{deg}$, providing a similar precision with the MTA technique. So, theoretical speaking, the MTA method, along with the MDD technique, may have the ability to measure the axial orientation of a certain flux rope more accurately than the traditional MVAbased methods. In order to estimate their precision beyond a theoretical point of view, it is interesting to apply the method to a real Cluster event, as will be done in the following section.

\section{Applications}

On 26 January 2001, several flux rope events were observed by Cluster satellites (e.g. Phan et al., 2004; Pu et al., 2005) when they were traveling outbound in the northern high-latitude regions from the magnetosphere to the magnetosheath, with the spacecraft separation of $\sim 600 \mathrm{~km}$. Three of those flux rope orientations obtained by MDD analysis were calculated and listed in Table 1 of Shi et al. (2005).

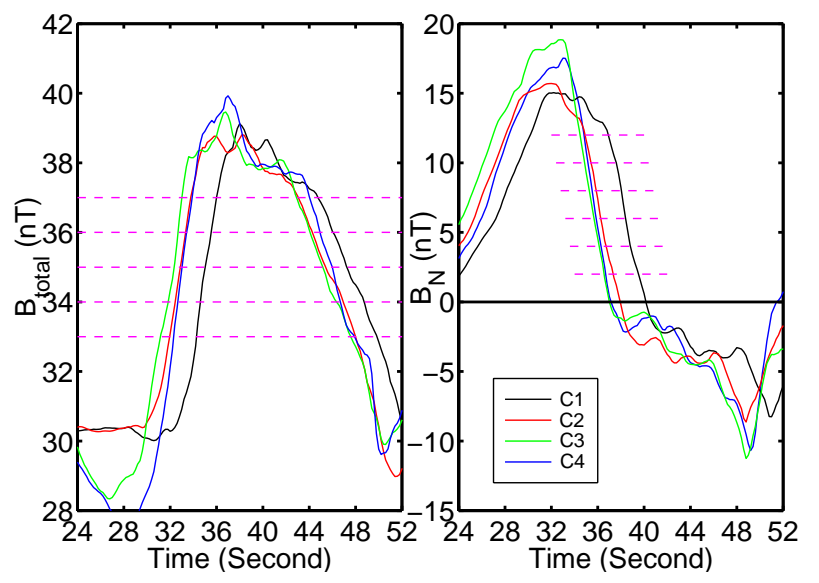

Fig. 3. A flux rope structure observed by FGM/Cluster on 26 January 2001, 11:10:24 UT to 11:10:52 UT. (Left panel) Magnetic strength, (right panel) $B_{N}$ components, as a function of time (both 4 -s sliding averages at 0.2 -s resolution).

As a comparison, the MTA technique is applied to analyze these flux ropes, using magnetic field data (FGM data) (Balogh et al., 1997). One of the events, in the time interval of 11:10:24 UT-11:10:52 UT, is shown in Fig. 3: the left panel represents the magnetic magnitude $|B|$ and the right panel is the $\mathrm{N}$ component of the magnetic field $B_{N}$. The $\mathrm{N}$ direction, being calculated as $(0.63,0.33,0.70)$ by Pu et al. (2005), using minimum variance analysis, can be considered as the normal direction of the magnetopause during this time period. The $|B|$ enhancement, along with the bipolar signature of $B_{N}$, clearly indicates a flux rope structure (Russell and Elphic, 1978).

In order to eliminate the undesirable high-frequency fluctuations in applying the Triangulation method (as the first step of MTA), a procedure of a sliding average is required (Haaland et al., 2004a). Here we use a sliding window of $4 \mathrm{~s}$, with a time resolution of $0.2 \mathrm{~s}$, and a apply linear interpolation between each two consecutive measurements.

Then we can select five magnetic field values $(33 \mathrm{nT}$, $34 \mathrm{nT}, 35 \mathrm{nT}, 36 \mathrm{nT}$ and $37 \mathrm{nT}$ ) as the signals, shown as magenta dashed lines in the left panel of Fig. 3, and correspondingly obtain 10 normal directions (5 for arriving and 5 for departure) using the timing method. The 10 directions, listed in the upper panel of Table 1, thus lead us to the calculation of three eigenvalues and their corresponding three eigenvectors. The eigenvector $(-0.136,0.991,0.002)$ with the smallest eigenvalue $\left(\lambda_{2} / \lambda_{3}=21.7\right)$ should be, in principle, perpendicular to all of the 10 normals, and could thus be treated as the estimated flux rope orientation.

The set of normals and eigenvectors are also displayed in the left GSE box of Fig. 4, as 10 blue lines and 3 red lines, respectively. The green circle, defined by the two eigenvectors with relatively larger eigenvalues, can be 

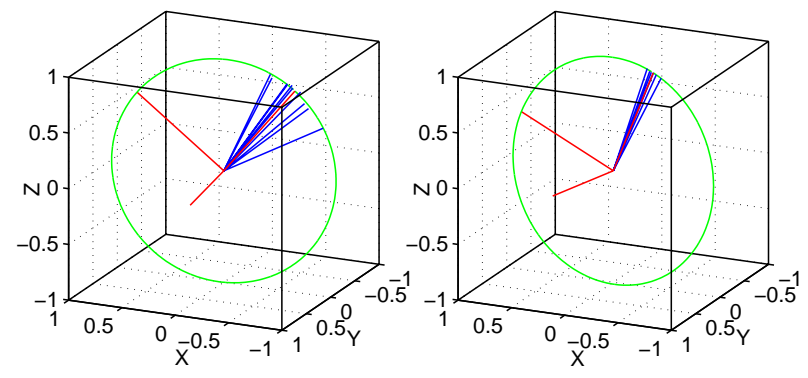

Fig. 4. The MTA analysis on the flux rope event during 11:10:24 UT to 11:10:52 UT. In the left box, the magnetic strength is selected as the signal, while in the right one, the $B_{N}$ component is selected. In either of the boxes, the blue lines show the normal directions obtained by the Triangulation method as the first step of MTA, and the red lines are the three eigenvectors of the corresponding matrix. The green circle represents the cross-section plane of the flux rope, basically containing all of the blue lines, while the red line which is perpendicular to the green circle suggests the flux rope orientation.

treated as the cross-section plane of the flux rope, and basically contain all of the 10 normals. The third red line, $(-0.136,0.991,0.002)$, being treated as the orientation of the flux rope, can be clearly seen to be perpendicular to the cross-section plane (and therefore to the 10 normals).

Based on the 2-D property of the flux rope model, the gradient of $B_{N}$ would also be within the cross-section plane. So the normal directions of all the $B_{N}$ contour planes are also perpendicular to the flux rope orientation. Therefore, we can select $B_{N}$ as the signal (instead of $|B|$ ) to perform the MTA technique, as another test of MTA validity. $\operatorname{Six} B_{N}$ are selected ( $2 \mathrm{nT}, 4 \mathrm{nT}, 6 \mathrm{nT}, 8 \mathrm{nT}, 10 \mathrm{nT}$ and $12 \mathrm{nT})$, also shown as magenta dashed lines in the right panel of Fig. 3, and the resulting normals are listed in the bottom panel of Table 1.

The six normals, along with the three corresponding eigenvectors, are shown in the right GSE box of Fig. 4, with the same format as the left one. Although they are shown to be very close to each other, they are still well confined in a plane with the normal to be $(0.117,0.992,0.051)$. This direction, also being treated as the flux rope orientation, is pretty similar to the one calculated before, which in some sense confirms the reliability and consistency of the MTA technique.

The other two events listed in Shi et al. (2005) are also recalculated using the MTA technique, shown in Table 2, along with the one we displayed before. Here $\lambda_{2}$ and $\lambda_{3}$ denote the intermediate and minimum eigenvalues of MTA, suggesting the third row $\lambda_{2} / \lambda_{3}$ to be an indicator of the MTA estimating precision. As a comparison, the resulting orientations obtained by some other methods, including MDD and CMVA, are also listed.

It can be clearly seen that the directions calculated by MTA technique are pretty close to those obtained by MDD, with a deviation between them of around $10 \mathrm{deg}$. The sim-
Table 2. MTA results of three flux ropes observed on 26 January 2001, along with the comparison with other methods.

\begin{tabular}{cccc}
\hline Time interval(UT) & Methods & Flux rope orientation & $\lambda_{2} / \lambda_{3}$ \\
\hline 10:54:12-10:54:32 & MTA & $(-0.1450 .983-0.112)$ & 22.2 \\
& MDD & $(-0.2160 .942-0.255)$ & \\
& CMVA & $(-0.2760 .821-0.500)$ & \\
\hline $11: 10: 24-11: 10: 52$ & MTA & $(-0.1360 .9910 .002)$ & 21.7 \\
& MDD & $(-0.2470 .939-0.239)$ & \\
& CMVA & $(-0.7230 .638-0.267)$ & \\
\hline $11: 30: 40-11: 31: 20$ & MTA & $(0.3400 .935-0.099)$ & 3.01 \\
& MDD & $(-0.2950 .920-0.257)$ & \\
& CMVA & $(-0.6830 .731-0.012)$ & \\
\hline
\end{tabular}

ilarity between them thus suggests the validity of the MTA method.

On the other hand, the CMVA results show relatively larger deviations, especially in the second and third cases. This is mainly caused by the error produced in the calculation of electric current. The typical $|\nabla \cdot \boldsymbol{B}| /|\nabla \times \boldsymbol{B}|$ value of $\sim 0.4$, indicating the inaccuracy of the electric current obtained, might strongly reduce the reliability of the CMVA method in these cases.

It should also be pointed out that the value of $\lambda_{2} / \lambda_{3}$ is as small as 3.01 in the last case, implying an uncertainty in determining the flux rope orientation. The uncertainty can be probably explained by the traversing path of Cluster to be too close to the flux rope center. Actually, in this case, the resulting deviations between MTA and MDD are relatively larger than the other two.

\section{Conclusions}

We have introduced another method called the MTA technique, to find out the axial orientation of a certain flux rope by a 4 -spacecraft constellation. Basically, the error range of the MTA technique critically depends on both the satellite separation distance and the constellation path via the flux rope. In spite of these, the MTA has proved itself to be an accurate method in a theoretical way. Also, the Cluster data has been used as an application of the MTA technique. Selecting both $|B|$ and $B_{N}$ as the signal, similar resulting orientations are obtained, suggesting the reliability of MTA. Comparisons with other methods are also made to test the precision of the technique. So, in principle, the MTA technique is providing another way to explore the flux rope structures, especially in those cases when other methods are invalid. In addition, the method may become even more accurate in some future missions with more than 4 satellites. 


\section{Appendix A}

\section{Error estimation for random errors}

The random errors of $N^{(m)}$, either caused by the measurement inaccuracy or by the quantization effects of the Triangulation method, can be treated as a set of errors being independent from each other and having identical probability distributions (independent, identical distributions, denoted as iid), which satisfy the assumption in Khrabrov and Sonnerup (1998a) (hereinafter as paper 1). Within this framework, the data series appear as one of the possible fluctuating states of the normal vector pattern.

So we can directly follow the procedure of paper 1, however, we do not mean to repeat the lengthy manipulations. Instead, we will mainly emphasize the difference between them, to obtain the final result of the MTA error range.

The minimization method applied in MTA would produce a symmetric matrix

$L_{\mu \nu}=\left\langle N_{\mu}^{(m)} N_{\nu}^{(m)}\right\rangle$

in comparison with the matrix in MVA

$M_{\mu \nu}=\left\langle B_{\mu}^{(m)} B_{v}^{(m)}\right\rangle-\left\langle B_{\mu}^{(m)}\right\rangle\left\langle B_{v}^{(m)}\right\rangle$,

where the brackets \langle\rangle represent the averaging process over the measured data set.

Note that another averaging operation, denoted by double brackets $\langle\langle\rangle$, means the average over the ensemble of all possible fluctuating states. And certainly the ensemble average, $\langle\langle\rangle\rangle$, and the average over the data in a given state, \langle\rangle , can be interchanged, i.e. $\langle[\langle\langle\rangle\rangle]\rangle=\langle\langle[\langle\rangle]\rangle\rangle$.

In order to depict a certain fluctuating state, the vector $N^{(m)}$ can be expressed as $N^{*(m)}+n^{(m)}$, where the asterisk means the signal and $\boldsymbol{n}$ is the noise with an iid distribution. Some properties of the iid distribution can be listed below:

$\left\langle\left\langle\boldsymbol{n}^{(\boldsymbol{m})}\right\rangle\right\rangle=0, \quad m=1,2 \ldots M$.

Equation (4) of paper 1 suggests that the mean value of the errors is zero, if taken over all of the fluctuating states. Another property is the one in Eq. (5) of paper 1:

$\left\langle\left\langle n_{\mu}^{(m)} n_{\nu}^{(k)}\right\rangle\right\rangle \equiv S_{\mu \nu} \delta_{m k}$,

where $S_{\mu \nu}$ is independent of $m$ and $k$, and stands for the second-order terms of the noise.

Now the symmetric matrix $L_{\mu \nu}$ can be turned to

$$
\begin{aligned}
L_{\mu \nu} & =\left\langle\left(N_{\mu}^{*(m)}+n_{\mu}^{(m)}\right)\left(N_{\nu}^{*(m)}+n_{\nu}^{(m)}\right)\right\rangle \\
& =L_{\mu \nu}^{*}+\left\langle n_{\mu}^{(m)} n_{\nu}^{(m)}\right\rangle+\left\langle n_{\mu}^{(m)} N_{\nu}^{*(m)}\right\rangle+\left\langle N_{\mu}^{*(m)} n_{\nu}^{(m)}\right\rangle .
\end{aligned}
$$

Then the ensemble average can be taken on both sides. Based on the properties of Eqs. (A1) and (A2), the last two terms in the right sides of Eq. (A3) can be neglected:

$$
\left\langle\left\langle L_{\mu \nu}\right\rangle\right\rangle=L_{\mu \nu}^{*}+\left\langle\left\langle\left[\left\langle n_{\mu}^{(m)} n_{\nu}^{(m)}\right\rangle\right]\right\rangle\right\rangle=L_{\mu \nu}^{*}+S_{\mu \nu}
$$

which would replace Eq. (8) of paper 1 in our procedure, and Eq. (11) of paper 1 would be correspondingly switch to

$L_{i j}^{*}+S_{i j}=\delta_{i j}\left\langle\left\langle\lambda_{i}\right\rangle\right\rangle$

in the coordinate system of $\langle\langle\mathbf{L}\rangle\rangle$ eigenbasis, and the components would be denoted by the subscripts $i$ and $j$.

The next step would be connecting the fluctuations of eigenvectors $\boldsymbol{x}_{i}$ and eigenvalues $\lambda_{i}$ to the noise in a given fluctuating state, by linearizing the eigenproblem. As is shown in paper 1 (Eq. 12), the component of $\boldsymbol{\Delta} \boldsymbol{x}_{\boldsymbol{i}}$ along $\left\langle\left\langle\boldsymbol{x}_{\boldsymbol{j}}\right\rangle\right\rangle$ can be expressed as:

$$
\Delta x_{i j}=-\Delta x_{j i}=\frac{\Delta L_{i j}}{\left\langle\left\langle\lambda_{i}\right\rangle\right\rangle-\left\langle\left\langle\lambda_{j}\right\rangle\right\rangle} \quad i \neq j
$$

which suggests the role of the certain fluctuating state, rotating $\boldsymbol{x}_{\boldsymbol{i}}$ at an angle of $\Delta x_{i j}$ toward $\boldsymbol{x}_{\boldsymbol{j}}$, in radians.

So, in order to obtain the angular error of the MTA eigenvectors, the variance of $\mathbf{L}$ should be first calculated as the following:

$$
\begin{aligned}
\left\langle\left\langle\left(\Delta L_{i j}\right)^{2}\right\rangle\right\rangle \equiv & \left\langle\left\langle\left(L_{i j}-\left\langle\left\langle L_{i j}\right\rangle\right\rangle\right)^{2}\right\rangle\right\rangle \\
= & \left\langle\left\langle\left\{\frac{1}{M} \sum_{m=1}^{M}\left(N_{i}^{*(m)}+n_{i}^{(m)}\right)\left(N_{j}^{*(m)}+n_{j}^{(m)}\right)\right.\right.\right. \\
& \left.\left.\left.-\frac{1}{M} \sum_{k=1}^{M}\left(N_{i}^{*(k)} N_{j}^{*(k)}+\left\langle\left\langle n_{i} n_{j}\right\rangle\right\rangle\right)\right\}^{2}\right\rangle\right\rangle .
\end{aligned}
$$

Equation (A7) can be expressed as the sum of a set of terms, each of which is proportional to the first, second, third and fourth moment of $\boldsymbol{n}$. As we know from Eq. (A1), the first order terms have the value of zero. Furthermore, if we assume that the noise is smaller than the signal, the third and fourth terms can be also neglected. Equations (A2) and (A4) can be used in the next manipulation, and Eq. (A7) turns to:

$\left\langle\left\langle\left(\Delta L_{i j}\right)^{2}\right\rangle\right\rangle=\frac{1}{M}\left(L_{i i}^{*} S_{j j}+L_{j j}^{*} S_{i i}+2 L_{i j}^{*} S_{i j}\right)$.

The combination of Eq. (A6) and Eq. (A8) thus leads to the angular error of the eigenvectors:

$$
\begin{aligned}
\Delta \phi_{i j} & \equiv \sqrt{\left\langle\left\langle\left(\Delta x_{i j}\right)^{2}\right\rangle\right\rangle}=\sqrt{\frac{\left\langle\left\langle\left(\Delta L_{i j}\right)^{2}\right\rangle\right\rangle}{\left(\left\langle\left\langle\lambda_{i}\right\rangle\right\rangle-\left\langle\left\langle\lambda_{j}\right\rangle\right\rangle\right)^{2}}} \\
& =\sqrt{\frac{L_{i i}^{*} S_{j j}+L_{j j}^{*} S_{i i}+2 L_{i j}^{*} S_{i j}}{M\left(\left\langle\left\langle\lambda_{i}\right\rangle\right\rangle-\left\langle\left\langle\lambda_{j}\right\rangle\right\rangle\right)^{2}}} .
\end{aligned}
$$

Consider Eq. (A5) and neglect the higher order terms, Eq. (A9) turns to:

$\Delta \phi_{i j}=\sqrt{\frac{\left\langle\left\langle\lambda_{i}\right\rangle\right\rangle S_{j j}+\left\langle\left\langle\lambda_{j}\right\rangle\right\rangle S_{i i}}{M\left(\left\langle\left\langle\lambda_{i}\right\rangle\right\rangle-\left\langle\left\langle\lambda_{j}\right\rangle\right\rangle\right)^{2}}}$.

Specifying $i=3$ with $j=1$ or 2 , and based on the fact that the eigenvalue $\lambda_{3}$ is entirely due to the noise, Eq. (A10) then becomes

$\Delta \phi_{3 j}=\sqrt{\frac{\lambda_{3} \lambda_{j}}{M\left(\lambda_{3}-\lambda_{j}\right)^{2}}}$. 
In arriving at this result, $S_{33}$ is replaced by $\lambda_{3}$ and we further assume $S_{j j}$ to also be of order of $\lambda_{3}$, so that $\lambda_{3} S_{j j}$ would be of a higher order and could be thus neglected, (see paper 1 for more detail).

So the random angular errors of the MTA flux rope orientation can be obtained, as the function of the three eigenvalues and also the number of normal vectors.

Acknowledgements. The authors thank H. Zhang and C.-J. Xiao for many helpful discussions. This work is supported by NSFC project 40390152 and 40528005 . The research was initiated during a visit by X.-Z. Zhou to Boston University in Fall/Winter 2004, supported by NASA grant NAG5-10108.

Topical Editor I. A. Daglis thanks C. J. Farrugia and another referee for their help in evaluating this paper.

\section{References}

Balogh, A., Dunlop, M. W., Cowley, S. W. H., Southwood, D. J., Thomlinson, J. G., Glassmeier, K.-H., Musmann, G., Lühr, H., Buchert, S., Acuña, M. H., Fairfield, D. H., Slavin, J. A., Riedler, W., Sachwingenschuh, K., and Kivelson, M. G.: The Cluster Magnetic Field Investigation, Space Sci. Rev., 79, 65-91, 1997.

Dunlop, M. W., Southwood, D. J., Glassmeier, K. H., and Neubauer, F. M.: Analysis of multipoint magnetometer data, Adv. Space Res., 8(9), 273-277, 1988.

Elphic, R. C. and Russell, C. T.: Magnetic Flux ropes in the Venus ionosphere: Observations and models, J. Geophys. Res., 88, 5872, 1983.

Elphic, R. C. and Southwood, D. J.: Simultaneous measurements of the magnetopause and flux transfer events at widely separated sites by AMPTE UKS and ISEE 1 and 2, J. Geophys. Res., 92, 13 666-13 672, 1987.

Farrugia, C. J., Elphic, R. C., Southwood, D. J., and Cowley, S. W. H.: Field and flow perturbations outside the reconnected field region in flux transfer events: Theory, Planet. Space Sci., 35(2), 227-240, 1987.

Haaland, S., Sonnerup, B. U. O., Dunlop, M. W., Balogh, A., Georgescu, E., Hasegawa, H., Klecker, B., Paschmann, G., PuhlQuinn, P., Rème, H., Vaith, H., and Vaivads, A.: Four-spacecraft determination of magnetopause orientation, motion and thickness: comparison with results from single-spacecraft methods, Ann. Geophys., 22, 1347-1365, 2004a.

Haaland, S., Sonnerup, B. U. O., Dunlop, M. W., Georgescu, E., Paschmann, G., Klecker, B., and Vaivads, A.: Orientation and motion of a discontinuity from Cluster curlometer capability: Minimum variance of current density, Geophys. Res. Lett., 31, L10 804, doi:10.1029/2004GL020,001, 2004b.

Hau, L. N. and Sonnerup, B. U. O.: Two-dimensional coherent structures in the magnetopause: Recovery of static equilibria from single-spacecraft data, J. Geophys. Res., 104, 6899-6918, 1999.

Hu, Q. and Sonnerup, B. U. O.: Reconstruction of magnetic clouds in the solar wind: Orientations and configurations, J. Geophys. Res., 107 (A7), doi:10.1029/2001JA000293, 2002.

Khrabrov, A. V. and Sonnerup, B. U. O.: Error estimates for minimum variance analysis, J. Geophys. Res., 103, 6641-6651, 1998a.
Khrabrov, A. V. and Sonnerup, B. U. O.: Orientation and motion of current layers: Minimization of the Faraday residue, J. Geophys. Res., 25, 2373-2376, 1998b.

Lepping, R. P., Jones, J. A., and Burgala, L. F.: Magnetic field structure of interplanetary magnetic clouds at 1 AU, J. Geophys. Res., 95, 11957-11 965, 1990.

Phan, T. D., Dunlop, M. W., Paschmann, G., Klecker, B., Bosqued, J. M., Rème, H., Balogh, A., Twitty, C., Mozer, F. S., Carlson, C. W., Mouikis, C., and Kistler, L. M.: Cluster observations of continuous reconnection at the magnetopause under steady interplanetary magnetic field conditions, Ann. Geophys., 22, 23552367, 2004.

Pu, Z. Y., Zong, Q. G., Fritz, T. A., Xiao, C. J., Huang, Z. Y., Fu, S. Y., Shi, Q. Q., Dunlop, M. W., Glassmeier, K. H., Balogh, A., Daly, P., Rème, H., Dandouras, J., Cao, J. B., Liu, Z. X., Shen, C., and Shi, J. K.: Multipule flux rope events at the high-latitude magnetopause: Cluster/Rapid observation on January 26, 2001, in: The Magnetospheric Cusps: Structure and Dynamics, edited by Fritz, T. A. and Fung, S. F., Springer, Netherlands, 191-212, 2005.

Russell, C. T. and Elphic, R. C.: Initial ISEE magnetometer results: Magnetopause observations, Space Sci. Rev., 22, 681-715, 1978.

Russell, C. T., Mellott, M. M., Smith, E. J., and King, J. H.: Multiple Spacecraft Observations of Interplanetary Shocks: Four Spacecraft Determination of Shock Normals, J. Geophys. Res., 88, 4739-4748, 1983.

Shi, Q. Q., Shen, C., Pu, Z. Y., Dunlop, M. W., Zong, Q.-G., Zhang, H., Xiao, C. J., Liu, Z. X., and Balogh, A.: Dimensional analysis of observed structures using multi-point magnetic field measurements: Application to Cluster, Geophys. Res. Lett., 32, L12 105, doi:10.1029/2005GL022454, 2005.

Siscoe, G. L., Davis Jr., L., Coleman Jr., P. J., Smith, E. J., and Jones, D. E.: Power Spectra and Discontinuities of the Interplanetary Magnetic Field: Mariner 4, J. Geophys. Res., 73, 61-82, 1968.

Sonnerup, B. U. O. and Cahill Jr., L. J.: Explorer 12 Observations of Magnetopause Current Layer, J. Geophys. Res., 73, 1757-1771, 1968.

Sonnerup, B. U. O. and Scheible, M.: Minimum and Maximum Variance Analysis, in: Analysis Methods for Multi-spacecraft Data, edited by: Paschmann, G. and Daly, P., ISSI/ESA, Netherlands, 185-220, 1998.

Sonnerup, B. U. O., Haaland, S., Paschmann, G., Lavraud, B., Dunlop, M. W., Rème, H., and Balogh, A.: Orientation and motion of a discontinuity from single-spacecraft measurements of plasma velocity and density: Minimum mass flux residue, J. Geophys. Res., 109, A03 221, doi:10.1029/2003JA010230, 2004.

Xiao, C. J., Pu, Z. Y., Ma, Z. W., Fu, S. Y., Huang, Z. Y., and Zong, Q. G.: Inferring of flux rope orientation with the minimum variance analysis technique, J. Geophys. Res., 109, A11218, doi:10.1029/2004JA010594, 2004.

Zong, Q.-G., Fritz, T. A., Spence, H., Dunlop, M., Balogh, A., Korth, A., Daly, P. W., Pu, Z. Y., and Rème, H.: Bursty Energetic Electrons in Flux Ropes in the Cusp Region, Planet. Space Sci., 51, 821-830, 2003. 